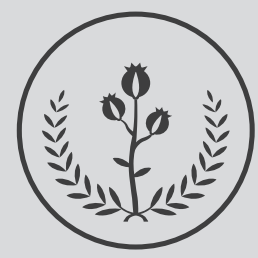

FUCS

\title{
Re

\section{Dosis aplicadas contra COVID-19 por laboratorio en las entidades territoriales de Colombia}

COVID 19 vaccine doses by pharmaceutical laboratory administered in the territorial entities of Colombia

a Ingeniero. Magister en Gestión de la Tecnología Educativa, Especialista en Administración de la Informática Educativa. Docente de matemáticas e Investigador, Secretaría de Educación de Soacha, Cundinamarca.

\section{R E S U M E N}

Introducción: en muchos países se están llevando a cabo campañas de vacunación masiva para prevenir la enfermedad por coronavirus 2019 (COVID-19); se necesitan con urgencia estimaciones de la eficacia de la vacuna para respaldar la toma de decisiones. Objetivo: presentar el avance de dosis completas por laboratorio en las diferentes entidades territoriales de Colombia al 24 de agosto 2021. Metodología: estudio de tipo transversal analítico diseñado de manera específica para recoger información sobre vacunas aplicadas en Colombia por laboratorio; como fuente de información se tomó el plan nacional de vacunación contra el COVID-19 de la página web del Ministerio de Salud y Protección Social. Resultados: las entidades territoriales con mayor número de dosis completas por laboratorio fueron: Bogotá (4'259.615), Antioquia (2'864.061), Valle del Cauca (2'004.655), Santander (1'289.588) y Cundinamarca (840.967), y las de menor: Vichada (12.784), Vaupés (13.754), Guainía (20.706), Guaviare (31.598) y Amazonas (54.385). Conclusión: el despliegue de las vacunas contra el COVID-19 en

\section{INFORMACIÓN DEL ARTÍCULO}

Historia del artículo:

Fecha recibido: agosto 30 de 2021

Fecha aceptado: septiembre 28 de 2021
Autor para correspondencia: Ing. Jorge Enrique Díaz Pinzón jediazp@unal.edu.co
DOI

10.31260/RepertMedCir.01217372.1278 
Colombia brinda una solución para apaciguar los efectos de la pandemia, pero también plantea retos. En Colombia existe el compromiso de garantizar que todos los sus habitantes tengan acceso a las vacunas de manera justa y equitativa.

Palabras clave: COVID-19, SARS-COV-2, pandemia, vacunas.

(C) 2021 Fundación Universitaria de Ciencias de la Salud - FUCS. Este es un artículo Open Access bajo la licencia CC BY-NC-ND (http://creativecommons.org/licenses/by-nc-nd/4.0/).

\section{ABS T RACT}

Introduction: mass vaccination campaigns to prevent coronavirus 19 disease (COVID 19) are being conducted in many countries; there is urgency of providing estimates on vaccine efficacy to support decision-making. Objective: to present the progression of completed vaccination schedules by type of vaccine in the different territorial entities in Colombia as of August 24 2021. Methodology: a cross-sectional study specifically designed to gather information on various pharmaceutical company vaccines administered in Colombia; data was obtained from the Ministry of Health and Social Protection webpage based on the COVID 19 national vaccination plan. Results: the territorial entities with the most completed schedules administered by type of vaccine were: Bogotá (4'259.615), Antioquia (2'864.061), Valle del Cauca (2'004.655), Santander (1'289.588) and Cundinamarca (840.967), and those with the least were: Vichada (12.784), Vaupes (13.754), Guainía (20.706), Guaviare (31.598) and Amazonas (54.385). Conclusion: the deployment of vaccines against COVID-19 in Colombia offers a solution to mitigate the effects of the pandemic, but also poses challenges. Colombia is committed to guarantee that everyone in the country has fair and equitable access to COVID 19 vaccines.

Key words: COVID-19, SARS-COV-2, pandemic, vaccines.

(C) 2021 Fundación Universitaria de Ciencias de la Salud - FUCS This is an open access article under the CC BY-NC-ND license (http://creativecommons.org/licenses/by-nc-nd/4.0/).

\section{INTRODUCCIÓN}

En muchos países hay campañas de vacunación masiva para prevenir la enfermedad por coronavirus 2019 (COVID-19). Se necesitan con urgencia estimaciones de la eficacia de la vacuna para respaldar la toma de decisiones. ${ }^{1}$

El 2 de diciembre 2020, se aprobó la primera vacuna probada en un gran ensayo clínico aleatorizado en el Reino Unido ${ }^{2,3}$, aunque algunos países comenzaron la aplicación antes de que los resultados clínicos estuvieran disponibles. Se han desarrollado y aprobado varias de ellas eficaces contra COVID-19 en un tiempo récord ${ }^{4-6}$ y son numerosas otras que se encuentran en las etapas finales de ensayos clínicos. ${ }^{7}$

Las vacunas son una medida esencial y se necesitan con urgencia para controlar la pandemia. ${ }^{8}$ La ideal sería la eficaz después de una o dos vacunaciones; protegería a las poblaciones objetivo, como los adultos mayores y las personas con comorbilidades, incluidas las personas inmunodeprimidas. ${ }^{9-11}$ El objetivo de esta investigación es presentar el avance de dosis completas por laboratorio en las diferentes entidades territoriales de Colombia al 24 de agosto 2021 .

\section{METODOLOGÍA}

Análisis de tipo transversal analítico diseñado concretamente para recoger información sobre vacunas administradas en Colombia. La fuente de información fue el plan nacional de vacunación contra el COVID-19 de la página web del Ministerio de Salud y Protección Social ${ }^{12}$, en el periodo comprendido entre el 17 de febrero 2021 a 21 de agosto 2021. Además, se manejaron medios matemáticos y estadísticos cotidianos para valorar los resultados de modo evidente. ${ }^{13-15}$

\section{RESULTADOS}

En la figura 1 podemos observar los valores de las dosis completas por laboratorio en las diferentes entidades territoriales de Colombia al 24 de agosto 2021. En ella se puede inferir que las de mayor número de dosis completas por laboratorio son: Bogotá (4'259.615), Antioquia (2'864.061), Valle del Cauca (2'004.655), Santander (1'289.588) y Cundinamarca (840.967), y las de menor son: Vichada (12.784), Vaupés (13.754), Guainía (20.706), Guaviare (31.598) y Amazonas (54.385). 


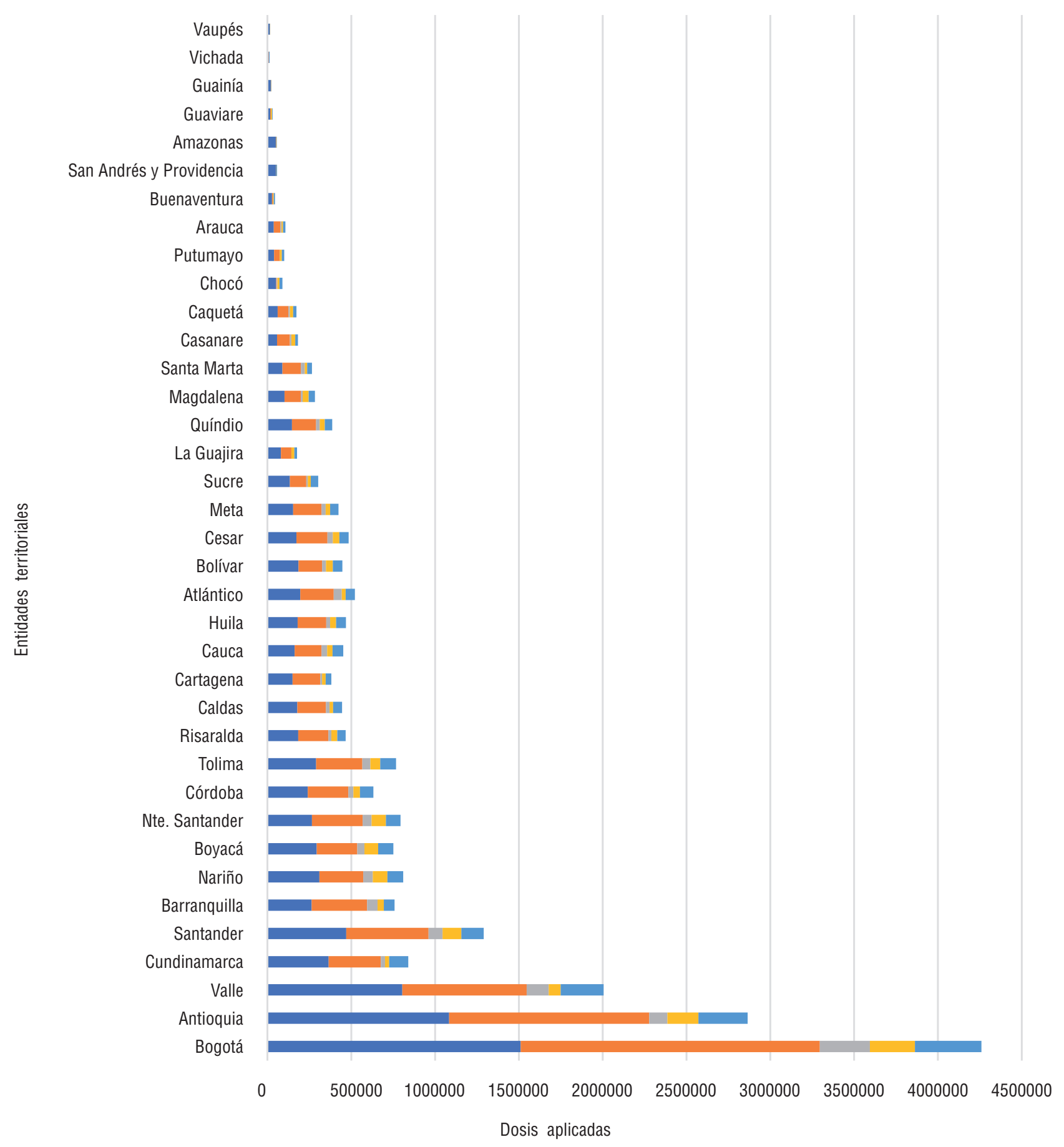

Sinovac $\square$ Pfizer $\square$ Moderna $\square$ Jansen $\square$ AstraZeneca

Figura 1. Dosis aplicadas por laboratorio en las entidades territoriales. Fuente: el autor.

En la figura 2 podemos considerar los valores de las dosis aplicadas por laboratorio contra SARS-COV-2 en las entidades territoriales de Colombia, a 24de agosto 2021. Se puede concluir que Sinovac y Pfizer tienen el mayor número de dosis aplicadas con 38\%, seguida de AstraZeneca $11 \%$, Jansen $7 \%$ y por último Moderna $6 \%$. 


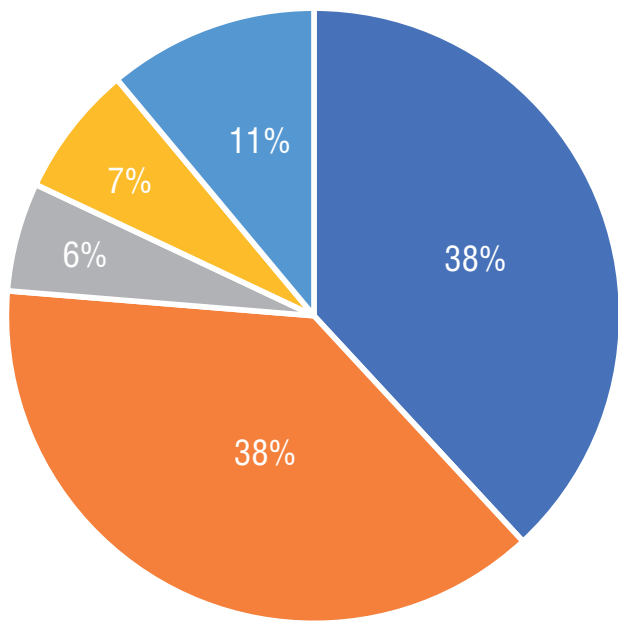

- Sinovac $\quad$ Pfizer $\square$ Moderna $\square$ Jansen $\quad$ AstraZeneca

Figura 2. Porcentaje de dosis aplicadas por laboratorio contra COVID-19. Fuente: el autor.

\section{CONCLUSIONES}

Se estableció que al 24 de agosto 2021 las entidades territoriales con mayor número de dosis completas por laboratorio fueron: Bogotá (4'259.615), Antioquia (2'864.061), Valle del Cauca (2'004.655), Santander (1'289.588) y Cundinamarca (840.967) y de menor: Vichada (12.784), Vaupés (13.754), Guainía (20.706), Guaviare (31.598) y Amazonas (54.385). Asimismo, se determinó que las vacunas Sinovac y Pfizer tienen el mayor número de dosis aplicadas con 38\%, seguidas de AstraZeneca $11 \%$, Jansen $7 \%$ y por último Moderna $6 \%$.

El despliegue de las vacunas contra el COVID-19 en Colombia brinda una solución para apaciguar los efectos de la pandemia, pero también plantea retos. Existe el compromiso de garantizar que todos los sus habitantes tengan acceso a las vacunas de manera justa y equitativa.

\section{REFEREN CI AS}

1. Jara A, Undurraga E, González C, Paredes F, Fontecilla T, et al. Effectiveness of an Inactivated SARS-CoV-2 Vaccine in Chile. N Engl J Med. 2021;385(10):875-884. doi: 10.1056/NEJMoa2107715

2. Polack FP, Thomas SJ, Kitchin N, Absalon J, et al. Safety and Efficacy of the BNT162b2 mRNA Covid-19 Vaccine. N Engl J Med. 2020;383(27):2603-2615. doi: 10.1056/NEJMoa2034577

3. Voysey M, Clemens SAC, Madhi SA, Weckx LY, Folegatti PM, et al. Safety and efficacy of the ChAdOxl nCoV-19 vaccine (AZD1222) against SARS-CoV-2: an interim analysis of four randomised controlled trials in Brazil, South Africa, and the UK. Lancet. 2021;39710269):99 -111. doi: 10.1016/S0140-6736(20)32661-1
4. Baden LR, El Sahly HM, Essink B, Kotloff K, Frey S, et al. Efficacy and Safety of the mRNA-1273 SARS-CoV-2 Vaccine. N Engl J Med. 2021;384(5):403-416. doi: 10.1056/NEJMoa2035389

5. Logunov DY, Dolzhikova IV, Shcheblyakov DV, et al. Safety and efficacy of an rAd26 and rAd5 vector-based heterologous primeboost COVID-19 vaccine: an interim analysis of a randomised controlled phase 3 trial in Russia. Lancet. 2021;397(10275):671681. doi: 10.1016/S0140-6736(21)00234-8

6. Tanne JH. Covid-19: FDA panel votes to approve Pfizer BioNTech vaccine. BMJ. 2020;371:m4799-m4799. doi: 10.1136/bmj.m4799

7. Zimmer C, Corum J, Wee SL. Coronavirus vaccine tracker [Internet]. New York Times; 2021 [citado junio de 10 2021]. Disponible en: https://www.nytimes.com/interactive/2020/science/coronavirusvaccine-tracker.html

8. Lurie, N., Saville, M., Hatchett, R. y Halton, J. Developing Covid-19 Vaccines at Pandemic Speed. N Engl J Med. 2020;382(21):19691973. doi: 10.1056/NEJMp2005630

9. Fidler S, Stöhr W, Pace M, Dorrell L, Lever A, Pett S, et al. Antiretroviral therapy alone versus antiretroviral therapy with a kick and kill approach, on measures of the HIV reservoir in participants with recent HIV infection (the RIVER trial): a phase 2, randomised trial. Lancet. 2020;395(10227):888 - 898. doi: 10.1016/S0140-6736(19)32990-3

10. T Hanke. Aiming for protective T-cell responses: a focus on the first generation conserved-region HIVconsv vaccines in preventive and therapeutic clinical trials. Expert Rev Vaccines. 2019;18(10):10291041. doi: 10.1080/14760584.2019.1675518

11. Mothe B, Manzardo C, Sanchez-Bernabeu A, Coll P, Morón-López $\mathrm{S}$, et al. Corrigendum to 'Therapeutic vaccination refocuses T-cell responses towards conserved regions of HIV-1 in early treated individuals (BCN 01 study)' EClinicalMedicine 11 (2019) 65-80. EClinicalMedicine. 2020;18:100250. doi: 10.1016/j. eclinm.2019.100250

12. Ministerio de Salud y Protección Social de Colombia. Plan de vacunación nacional contra COVID-19 [Internet]. Ministerio de Salud y Protección Social de Colombia; Colombia. 2021 [citado 10 de junio 2021]. Disponible en: https://www.minsalud.gov.co/ salud/publica/Vacunacion/Paginas/Vacunacion-covid-19.aspx

13. Díaz Pinzón JE. Estimación de la prevalencia del COVID-19 en Colombia. Repert Med Cir. 2020;29(Núm. Supl.1):99-102. https:// doi.org/10.31260/RepertMedCir.01217372.1115

14. Díaz Pinzón JE. Análisis de los resultados del contagio del COVID-19 respecto a su distribución geográfica en Colombia. Repert Med Cir. 2020;29(Núm. Supl.1):60-64. https://doi.org/10.31260/ RepertMedCir.01217372.1082

15. Díaz Pinzón JE. Dinámica y relación del contagio del COVID-19 después de iniciado el plan de vacunación contra el SARS-COV-2 en Colombia. Repert Med Cir. 2021;30(Núm. Supl.1):41-45. https://doi.org/10.31260/RepertMedCir.01217372.1227 